\title{
Light Extraction from Plasmonic Particles with Dielectric Shells and Overcoatings
}

\author{
Phillip Manley ${ }^{1,2}$, Frank Schmidt ${ }^{2}$, Martina Schmid ${ }^{1}$ \\ ${ }^{1}$ Helmholtz Zentrum Berlin für Materialien und Energie, NanooptiX, \\ Hahn-Meitner Platz 1, 14109 Berlin, Germany, \\ ${ }^{2}$ Zuse Institute Berlin, Takustraße 7, 14195 Berlin, Germany \\ phillip.manley@helmholtz-berlin.de
}

\begin{abstract}
We rigorously simulate light scattering via the FEM from core-shell plasmonic particles and plasmonic particles with an isolating overcoat, in order to recommend design principles for maximising plasmonic scattering gains.
\end{abstract}

\section{Core Shell Particles}

It has been shown that using metallic nanoparticles to scatter light into high angle modes can increase the absorption of solar cells [1]. For use in conventional photovoltaic systems several challenges need to be overcome to integrate metallic nanoparticles. Firstly they should either be electrically isolated or positioned so as to not act as recombination centers. Secondly for certain metals, high temperature photovoltaic deposition techniques, like those used for $\mathrm{Cu}(\mathrm{In}, \mathrm{Ga}) \mathrm{S}_{2}$ (CIGS), can lead to a diffusion of the metal into the absorbing layer which will have deleterious effects. It may be possible to achieve electrical and chemical isolation by the use of either metal core / dielectric shell particles or dielectric over-coating of deposited metallic nanoparticles [2]. This extra dielectric layer must be taken into account from the optical viewpoint; this work aims to elucidate the design principles leading to maximum light extraction from the aforementioned dielectrically isolated plasmonic systems.

The two most commonly used plasmonic materials for photovoltaic applications are Ag and Au. In this work we cover simulations of both materials. The most common dielectric material used in shell layers is $\mathrm{SiO}_{2}$ because it is non-absorbing, non-conducting and easy to coat onto a metallic particle. For back-reflector nanoparticles a transparent conducting oxide like tin doped indium oxide (ITO) can be used for the dual purpose of isolating the metallic nanoparticles and acting as a back contact to the solar cell.
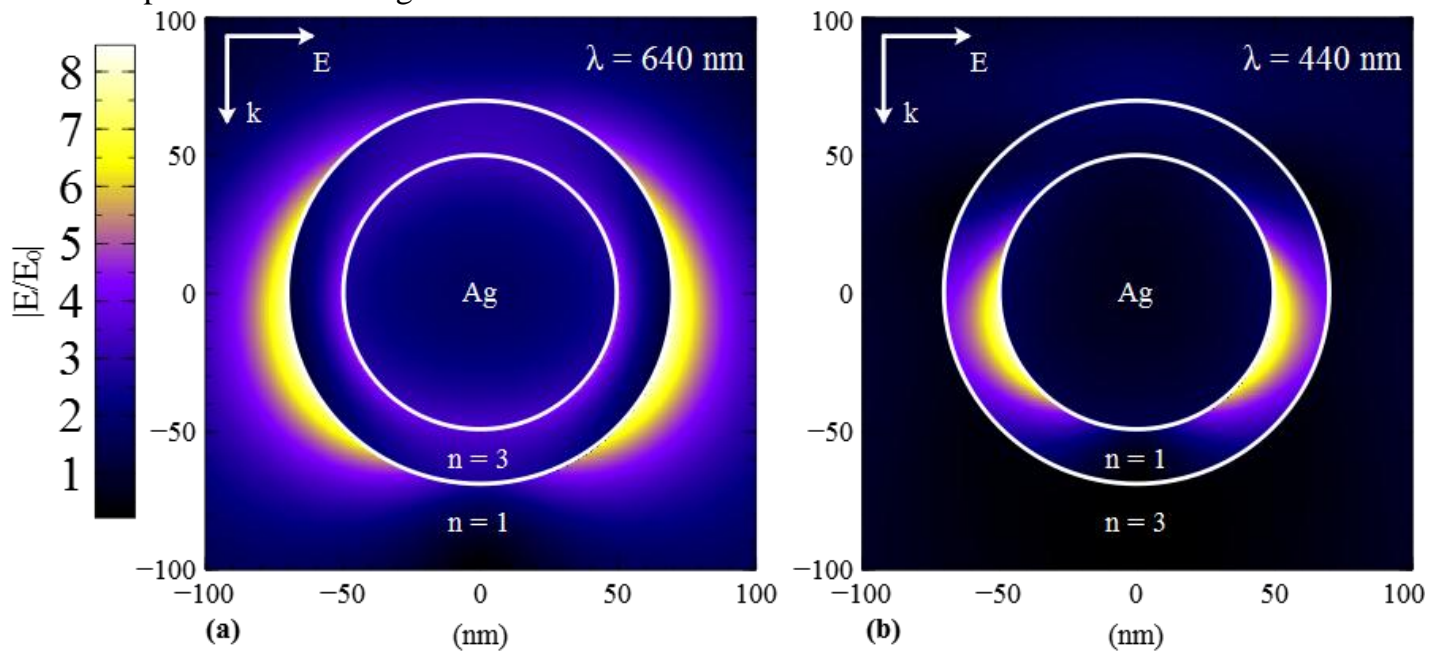

Fig. 1. The near field enhancement compared with the incident field for two different core shell particles. The core is a radius $50 \mathrm{~nm} \mathrm{Ag}$ spherical particle whose surface is shown by the inner white circle. The shell, whose surface is shown by the outer white circle, has a radius of 70 $\mathrm{nm}$. The optical constants for the shell are a constant refractive index of (a) 3 and (b) 1, whilst the outer medium has a refractive index of (a) 1 and (b) 3. The axis in the upper left shows the direction of the incident light and the polarization. The wavelength was selected to be at the plasmonic resonance of the system

We use the JCMwave finite element solver to solve Maxwell's equations for two different geometries [3]. The first geometry is the core-shell particle system. We begin with a radius $50 \mathrm{~nm}$ spherical $\mathrm{Ag}$ nanoparticle with a 20 $\mathrm{nm}$ thick spherical dielectric shell (N.B. radius of dielectric shell is $70 \mathrm{~nm}$ ). The core-shell particle is then surrounded by a different dielectric medium with perfectly matched layer (PML) boundary conditions in all 
directions. The two different dielectrics have different refractive indices (shell $n_{2}$ and surrounding medium $n_{3}$ ) which are constant with wavelength and both are assumed to be non-absorbing within the wavelength range of interest. The indices $n_{2}$ and $n_{3}$ are varied independently in order to understand which combinations lead to extraction of plasmonically scattered light.

Figure 1 shows the near fields for two combinations of $n_{2}$ and $n_{3}$. Part (a) shows the near field for the case of $n_{2}$ $=3$ and $n_{3}=1$. The resulting near field is almost totally located outside the shell meaning a large light extraction from the shell. This corresponds to a high scattering into the far field. Part (b) has the situation reversed with $n_{2}=1$ and $n_{3}=3$. This time the near field is concentrated entirely within the shell meaning a low level of light extraction. The resulting far field scattering is also very low.

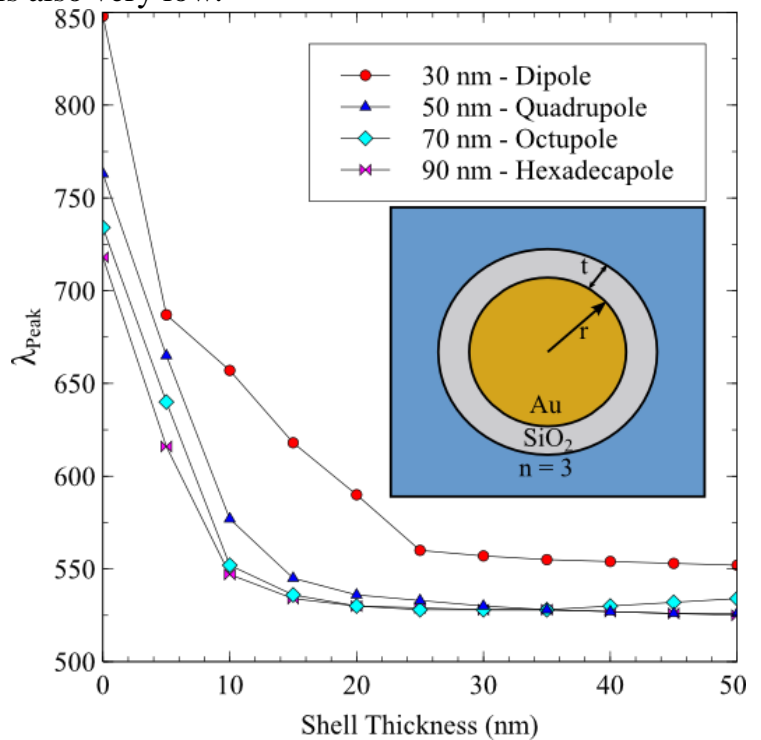

Fig. 2. The wavelength of the peak in absorption cross section for a Au sphere with a $\mathrm{SiO}_{2}$ shell surrounded by a homogeneous medium with refractive index 3 (as shown in the inset). The core radii are $30 \mathrm{~nm}$ (circles), $50 \mathrm{~nm}$ (triangles), $70 \mathrm{~nm}$ (diamonds) and $90 \mathrm{~nm}$ (ties). Only the position of the most strongly absorbing mode (e.g. dipole) is shown in each case.

These results are in contrast to the classical theory of waveguides in which light is trapped inside an optically dense medium surrounded by a less dense one. Our findings agree with the work of Beck et al. [4] who simulated $\mathrm{Ag}$ particles on top of a $\mathrm{Si}$ substrate with and without a $\mathrm{SiO}_{2}$ spacer layer. When including a thin spacer layer, which is analogous to the present work, they found a reduction of the total scattering in comparison to no spacer layer. In the case of plasmonic core shell particles, the shell should be optically denser than the surrounding medium for good light extraction. Further work aims to simulate high index dielectric shells as they will give better scattering responses.

The same geometry with different materials was investigated for the radius dependence of the dielectric shell. A Au core with a $\mathrm{SiO}_{2}$ shell surrounded by a homogeneous medium with refractive index of 3 was simulated. The core radius was varied between 30 and $90 \mathrm{~nm}$ and the shell thickness was varied simultaneously between 0 and $50 \mathrm{~nm}$. Figure 2 shows how the wavelength of the peak in the normalized absorption cross section changes with shell thickness. The values are normalized to the total core + shell radius. The absorption cross section was chosen because the scattering cross section also has components from the shell surface, whereas the absorption cross section only contains plasmonic effects. It can be seen that, for this example, the peak position converges to the value it has for a particle in homogeneous $\mathrm{SiO}_{2}$ after about $25 \mathrm{~nm}$. The exponential shift in peak position with changing shell thickness is due to the exponential decrease in near field intensity away from the metal/shell interface. The higher order modes decrease in intensity more rapidly moving away from the interface, explaining why the shift in the dipole mode is less rapid in comparison to the other modes.

The normalised scattering cross section, which is not shown here, reduces rapidly in intensity and is blueshifted out of the required wavelength even for small shell thicknesses $(5 \mathrm{~nm})$. This is to be expected as the results from the previous section showed that a $\mathrm{SiO}_{2}(\mathrm{n} \approx 1.5)$ shell embedded inside a high index material will not scatter efficiently. The implication is that even for relatively thin shells, the shell must be more optically dense than the medium. Additionally the spectral position of the plasmon should be optimized for the shell and not for the surrounding medium. 


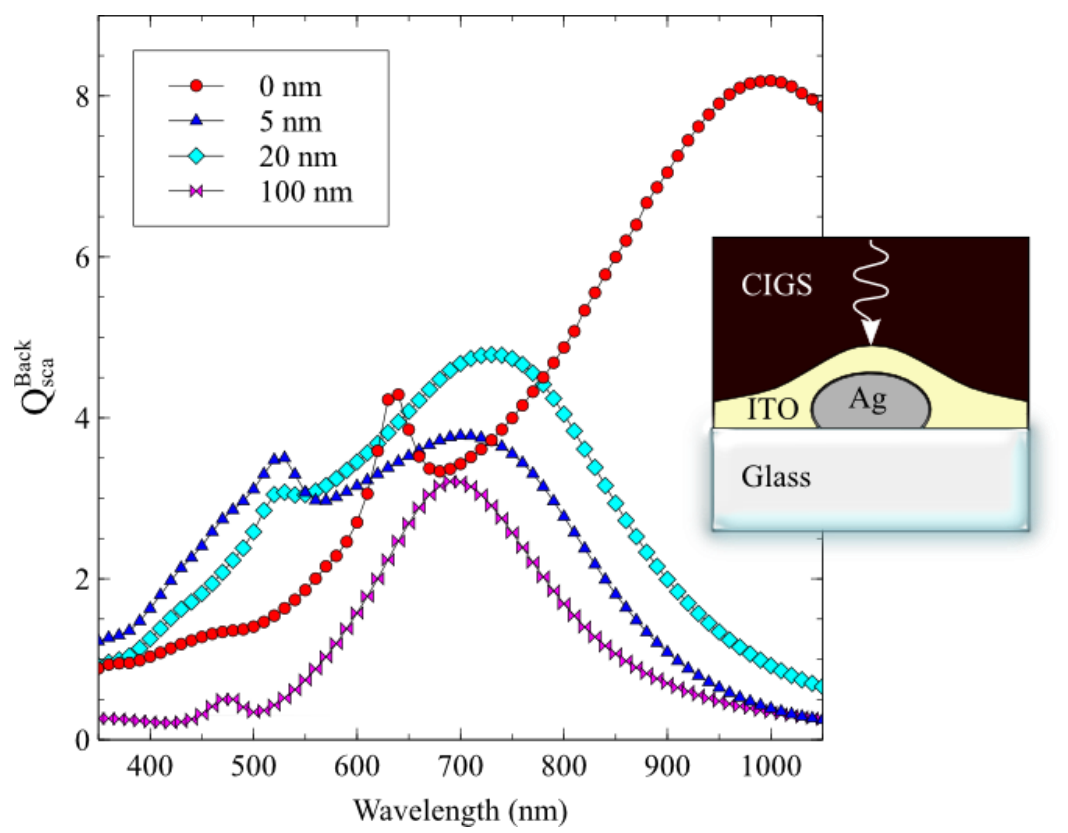

Fig. 3. The normalized back scattering cross section for a Ag nanoparticle at a glass / CIGS interface with different thicknesses of ITO overcoating (as shown in the inset). The thicknesses of the ITO overcoats are $0 \mathrm{~nm}$ (circles), $5 \mathrm{~nm}$ (triangles), $20 \mathrm{~nm}$ (diamonds) and $100 \mathrm{~nm}$ (ties). The wavelength range shown is the operational wavelength of a CIGS solar cell.

\section{Isolating Overcoat Layers}

The optical effect of isolating back reflecting oblate ellipsoidal Ag nanoparticles via an ITO overcoat was also investigated. The inset of Fig. 3 shows the simulation setup. Only the real part of the refractive index for CIGS was used so that light could be coupled in through the infinite CIGS layer. All boundaries of the computational domain were simulated using a PML. Due to simulating an isolated particle, the effects of waveguided modes will not be included in the simulation. However for a finite size distribution and randomized position and orientation of ellipsoidal nanoparticles, periodic effects like waveguide modes are not expected to play a significant role. Figure 3 gives spectra showing the amount of light scattered back into the CIGS layer for different ITO overcoat thicknesses. The scattered energy is normalized to the energy of the incoming light and to the ratio of particle cross sectional area to computational domain cross section area. The addition of a $5 \mathrm{~nm}$ overcoat strongly blueshifts and reduces in intensity the scattering peak in comparison to no overcoat. This is due to two effects combined. Firstly the particle having a lower scattering cross section at an ITO/glass interface compared to a CIGS/glass interface. Additionally there is a similar effect to the core shell particles from 1; the plasmonic field does not transfer well from a lower index medium to a higher one.

The total scattering increases as the thickness of the ITO layer is increased to $20 \mathrm{~nm}$. This is because as the incident field varies sinusoidally inside the ITO layer, moving the position of the particle inside the layer (or equivalently changing the thickness of the layer) will change the local intensity of light at the nanoparticle [5]. This highlights the need to simulate the entire solar cell device coherently in order that the local intensity of light at the nanoparticle is comparable to real world situations. Finally increasing the ITO thickness still further to $100 \mathrm{~nm}$ decreases the scattering significantly. This is due to absorption in the ITO layer which can be seen as the scattering is reduced more in the short wavelength which will be more strongly absorbed.

The authors would like to thank G. Yin for the optical constants used in the simulation of CIGS and ITO layers. The authors would like to acknowledge funding by the DFG (German Research Foundation) in the DFG research center MATHEON and support from the Initiative and Networking fund of the Helmholtz Association for the Young Investigator Group VH-NG-928.

1. Catchpole, K.R. and A. Polman, Plasmonic solar cells. Optics Express, 2008. 16(26): p. 21793-21800.

2. Schmid, M., et al., Stability of plasmonic metal nanoparticles integrated in the back contact of ultra-thin $\mathrm{Cu}(\mathrm{In}, \mathrm{Ga}) \mathrm{S}_{2}$ solar cells. Thin Solid Films, 2013. 527: p. 308-313.

3. Burger, S., et al. Finite-Element Simulations of Light Propagation through Circular Subwavelength Apertures. in SPIE Photonic Materials, Devices, and Applications III. 2009.

4. Beck, F.J., A. Polman, and K.R. Catchpole, Tunable light trapping for solar cells using localized surface plasmons. Journal of Applied Physics, 2009. 105: p. 114310.

5. Santbergen, R., S. Solntsev, and M. Zeman. Modelling Plasmonic Solar Cells. in $25^{\text {th }}$ EPVSEC. 2010. Valencia, Spain. 